Classification

Physics Abstracts

$07.80-87.15$

\title{
New possibilities in the observation of nucleic acids by electron spectroscopic imaging
}

\author{
Etienne Delain $\left({ }^{1}\right)$, Alain Fourcade $\left({ }^{1}\right)$, Bernard Révet $\left({ }^{1}\right)$ and Claudie Mory $\left({ }^{2}\right)$ \\ ( $\left.{ }^{1}\right)$ Laboratoire de Microscopie Cellulaire et Moléculaire, URA 147 du Centre National de la \\ Recherche Scientifique (CNRS), Institut Gustave Roussy, rue Camille Desmoulins, F-94805 \\ Villejuif Cedex, France \\ (2) Laboratoire de Physique des Solides associé au CNRS, Bâtiment 510, Université Paris Sud, \\ F-91405 Orsay Cedex, France
}

(Received May 25, 1992; accepted June 09, 1992)

\begin{abstract}
Résumé. - Les conditions d'utilisation de l'imagerie par spectroscopie de pertes d'énergies des électrons pour l'observation de molécules d'ADN adsorbées sur film de carbone sont décrites. Le microscope électronique Zeiss 902 avec spectromètre incorporé à la colonne permet de former des images selon des modes originaux, tel que le fond noir annulaire filtré en énergie. Les six différents modes de formation des images sont illustrés sur des préparations d'ADN coloré positivement ou métallisé, pour montrer les importantes différences de contrastes. Les très forts contrastes obtenus en fond noir annulaire ou spectroscopique à partir de préparations ne présentant que très peu de contraste en fond clair traditionnel sont très utiles pour l'observation de macromolécules biologiques. Les avantages de certains modes d'observation pour l'étude de différents types de préparations sont discutés.
\end{abstract}

\begin{abstract}
The conditions for the use of Electron Spectroscopic Imaging (ESI) in the observation of DNA molecules adsorbed on carbon film are described. The Zeiss 902 electron microscope with its built-in spectrometer allows original modes of imaging, such as energy-filtered annular dark-field. The six different modes of imaging are illustrated with either positively stained or metallized DNA preparations to show the resulting important differences in contrast. The very strong contrasts obtained in annular or spectroscopic dark-field modes on specimens presenting a very low contrast in traditional bright-field are very useful for the observation of biological macromolecules. The advantages of some observation modes for imaging different types of preparations are discussed.
\end{abstract}

\section{Introduction.}

One of the possibilities for studying the conformation of nucleic acids and their interactions with proteins is the visualization of these molecules using an electron microscope. Deoxyribonucleic acid (DNA), the support of heredity of living organisms, is a double-stranded molecule formed by two antiparallel chains of phosphate-sugar backbone bound by pairs of bases (adenine-thymine, guanine-cytosine) joined by hydrogen bonding inside the helix. Single-stranded DNA can be 
obtained by breaking these bonds, but the visualization of such molecules remains difficult to realize routinely, unless they are covered by proteins like cytochrome c. The distance between the base pairs is $3.4 \AA$, and each turn of the helix contains about 10 base pairs. The diameter of the helix is about $20 \AA$ and its length can be very long (about 1 meter for the human genome and $1 \mathrm{~mm}$ for that of a bacteria), or smaller and thus more accessible to a direct visualization in the electron microscope (a few $\mu \mathrm{m}$ for a virus or a bacteriophage genome and even less for the small pieces of DNA constructed by molecular biologists). Several methods for the visualization of DNA by electron microscopy have been proposed (reviewed in [1]) using different techniques for its spreading and either its staining or shadowing. The direct attachment of the DNA to a thin carbon film allows an easy observation in dark-field of the stained molecule [2] and will be illustrated here. Annular dark-field illumination provides highly contrasted images of the stained DNA filament and has been extensively used in our laboratory to study DNA and DNA-protein complexes involved in gene regulation and in physiological processes (reviewed in [3]).

DNA can be visualized by various procedures. Long DNA molecules are visible by UV light microscopy provided that they are stained with a fluorescent dye [4]. Radioactively labelled replicating DNA molecules can also be indirectly detected by autoradiography at the light microscope level [5]. Recently, near-field microscopes have been considered as susceptible to image DNA molecules at atomic resolution (reviewed in [6]), but this approach still needs additional research to obtain significant results, since the spreading of nucleic acid molecules for their visualization and even their sequencing by near-field microscopes still remains under investigation [7-10].

Electron microscopy is still the methodology which gives the widest possibilities for studying nucleic acids conformations. Besides the dark-field illumination procedures needed to get contrasted images of naked DNA molecules, new approaches are provided by the use of microscopes allowing Electron Energy Loss Spectroscopy (EELS) and Electron Spectroscopic Imaging (ESI). Only two types of commercially available microscopes allow such imaging modes: the Zeiss 902 with its built-in electron spectrometer [11] and the Scanning Transmission Electron Microscope (STEM, Vacuum generators HB-501) fitted with a magnetic sector.

Traditionally, dark-field modes (tilted or annular) are very efficient to visualize molecules either stained with uranyl salts or shadowed with tungsten-tantalum alloy $[12,13]$. They have been used in our laboratory for many biological applications on nucleic acids [3] or proteins [14]. Platinum, classically used for shadowing, makes deposits which diffract electrons and thus is not suitable for high resolution imaging of molecules. The problem of the thickness of this metal deposit has been the subject of many discussions since it greatly influences the resolution $[13,15]$.

In the Zeiss 902 electron microscope, the Castaing-Henry prism [16] allows direct imaging of the sample with energy-selected electrons, which are scattered elastically (deflected at wide angles without interaction with the electronic shell) or inelastically (either on an elemental-specific energy loss $\Delta E$ or not). In combination with the use of parallel (bright-field) or hollow-cone (darkfield) illumination, such an electron energy filtering microscope provides new types of imaging which have become routinely used in biological work on macromolecules. Besides the possibilities of observing in ESI large proteins negatively stained with uranyl acetate [17], applications for an optimal observation of DNA molecules have already been briefly presented [18] and are further developed in the present paper.

\section{Material and methods.}

BIOLOGICAL MATERIAL. - DNA molecules can be anyone used in molecular biology work. These molecules are usually linear or circular, either supercoiled or relaxed plasmids isolated 
from bacteria or purified genomes from viruses or bacteriophages. The DNA must be prepared and purified with great care to avoid the presence of proteins or any other contaminant. In the present work, the studied molecules are either the replicative form of the bacteriophage $\phi \times 174$ or the plasmid pBR322, both being circular and double-stranded. Our laboratory has also experience with the visualization of ribonucleic acid (RNA) molecules, either single- or double-stranded and of synthetic polynucleotides, with which conformations such as triple-stranded helices can also be obtained and observed.

SPECIMEN PREPARATION. - The observation of DNA molecules $20 \AA$ in diameter needs to bind them tightly to a support film as thin as possible in order to minimize its own contribution in any imaging mode. These supports are made of amorphous carbon deposited onto mica pieces by evaporating a thread of fibrous carbon from Balzers in a MED 010 evaporator. Modifications of the distance mica-source and of the evaporation conditions, allow to find the adequate thickness of the carbon film. These films are floated off from the mica by obliquely plunging the mica in water; the carbon film, floating at the surface of the water, is deposited on 600 mesh hexagonal copper grids by lowering the water level.

Three different methods were used for the attachment of the molecules to a support (Fig. 1).

A

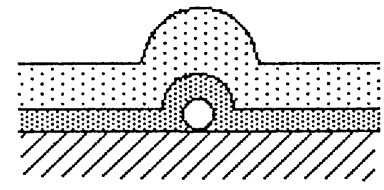

B

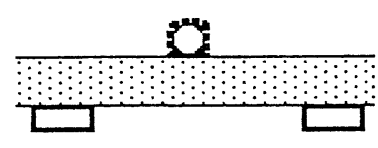

C
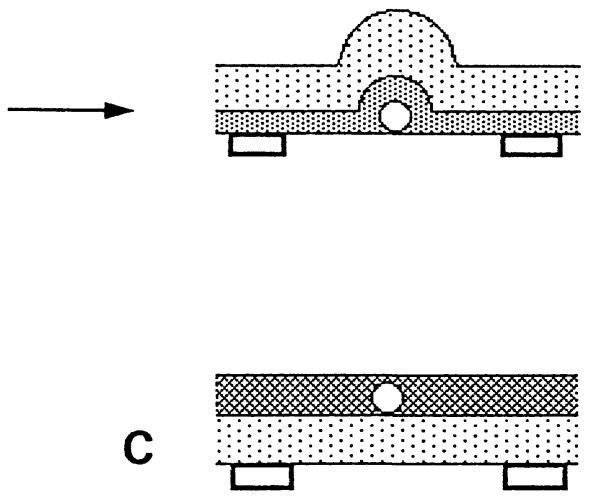

\begin{tabular}{|c|c|c|c|}
\hline (1) & Platinum-carbon shadowing & x & Uranyl acetate film \\
\hline$\vdots \vdots \vdots: \vdots$ & Carbon film & & Grid bars (schematic) \\
\hline DZA & Mica & 0 & Section of a DNA molecule \\
\hline
\end{tabular}

Fig. 1. - Schematic representation of the different modes of preparation described in the text. The DNA molecule and the different films are presented roughly at scale. A: Direct deposition of DNA molecules on a mica piece. Such a specimen is visualized in TEM as a replica obtained by carbon-coating the molecules shadowed with platinum-carbon. The arrow indicates that the replica is detached from the mica and deposited on a bare grid. B: Positive staining with uranium ions of a DNA molecule deposited on a carbon-coated grid. The uranium ions are bound to the negative phosphate groups at the periphery on the molecule, even, if the result is quite different after electron irradiation. C: Negative staining of a DNA molecule. The DNA adsorbed onto an hydrophilic carbon film deposited on a grid, is embedded in a film of uranyl acetate which makes an amorphous film of electron dense material protecting the molecules. 
- The first one (method A), used to prepare the shadowed molecules shown in figure 2, consists of a direct adsorption of the DNA molecules onto the surface of cleaved mica, in order to observe them also in Scanning Tunnelling (STM) or Atomic Force Microscopes (AFM). Shortly, the adsorption needs the presence of $\mathrm{Mg}^{2+}$ cations in the DNA solution and an uranyl acetate washing to promote the binding of DNA to the mica surface [10]. For Transmission Electron Microscopy (TEM), a platinum-carbon replica of the DNA molecules was prepared and detached from the mica. Shadowings are made in a Balzers MED 010 equipped with an electron gun to evaporate carbon-platinum rods or tungsten-tantalum alloy. They are usually made at angles of about $7^{\circ}$ during the rotation of the specimen holder. They are then stabilized by a thin layer of carbon. No thickness monitor being present in this evaporator, its control is made by checking with eye the density of the metal deposit on a white porcelain chip.

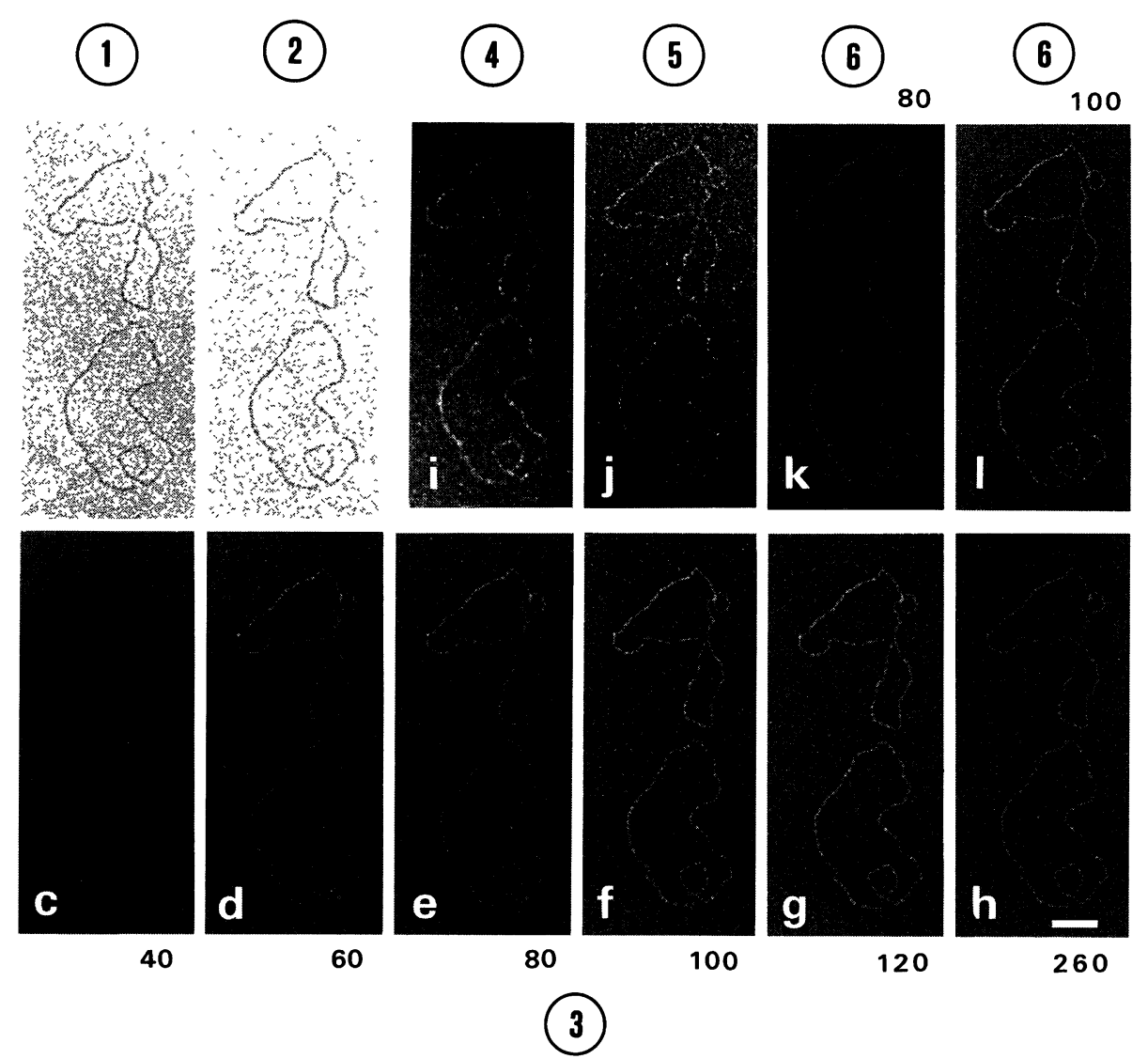

Fig. 2. - Platinum-carbon replica of two circular double-stranded DNA molecule (plasmid pBR322, contour length $1.48 \mu \mathrm{m}$ ) adsorbed onto mica and observed in the six different modes of imaging with a Zeiss 902, defined in table I. All pictures are printed so that they keep the original differences of contrast. The imaging modes and the $\Delta E$ values are indicated for each image or set of images. The observed variations in contrast are discussed in the text. Bar $=0.1 \mu \mathrm{m}$.

- In the second one (method B), used to prepare the positively stained molecules displayed in figures 3 and $4 a$ and $4 b$, grids covered with a thin carbon film are activated by a glow discharge in 
the presence of pentylamine according to Dubochet et al. [2] and used within a few hours. Five $\mu 1$ of a solution of $0.5 \mu \mathrm{g} / \mathrm{ml}$ of DNA are deposited onto the grid for one minute, then washed with an aqueous solution of uranyl acetate or thorium nitrate at concentrations ranging from $2 \%$ to as low as $10^{-4} \%$. The grid is then drained with a filter paper and let to dry in air. It has been observed that the spreading out of the DNA molecules requires both the activation of the carbon surface with pentylamine, which covers the carbon with $\mathrm{NH}^{+}$sites active to bind the DNA which is globally negatively charged and also the use of heavy metals such as actinides, which probably provide a definite precipitation and adsorption of the DNA to the positively charged carbon film. Various lanthanides salts have also been used to test the capacity of these ions to help the spreading out of the DNA molecules.

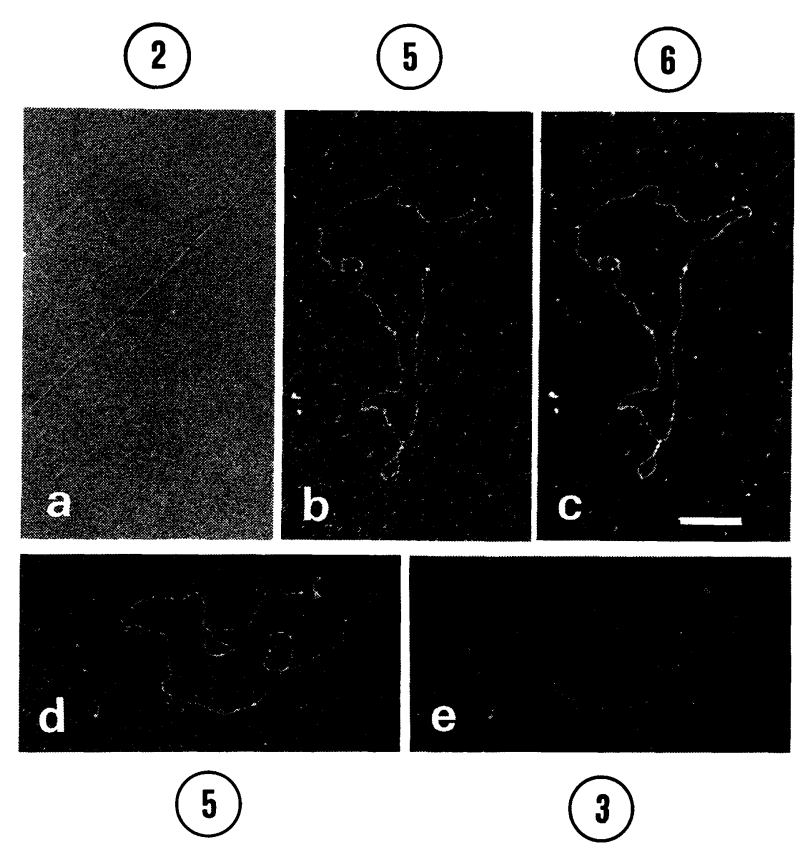

Fig. 3. - Circular double-stranded DNA molecule from the bacteriophage $\phi$ X174 (replicative form, contour length $1.83 \mu \mathrm{m}$ ), adsorbed on a thin carbon film, positively stained with uranium (Figs. 3a-c) or thorium (Figs. 3d and 3e) and observed in different modes of imaging. The imaging modes are indicated for each set of images. For figures $3 \mathrm{c}$ and $3 \mathrm{e}, \Delta E=114$ and $95 \mathrm{eV}$, respectively. The molecule seen in bright-field (a) with a poor contrast is better percieved in dark-field (b). Spectroscopic dark-field (c and e) shows a smoother background than the elastic annular dark-field ( $b$ and d). Molecules stained with uranium (a-c) or thorium (d and e) are equally well contrasted. Bar $=0.1 \mu \mathrm{m}$.

- The third one (method $C$ ) is called negative staining because the molecules are embedded in a film of stain, the thickness of which is equivalent to that of the molecules (Figs. 4c and 4d). The carbon-coated grids must be treated by a glow discharge in air (residual pressure $8 \times 10^{-1} \mathrm{mbar}$ ) to make them hydrophilic [19]. A few $\mu$ l of the solution of DNA or DNA-protein complex are let one minute for adsorption and the excess of solute washed out with $2 \%$ aqueous uranyl acetate, drained and air dried. 


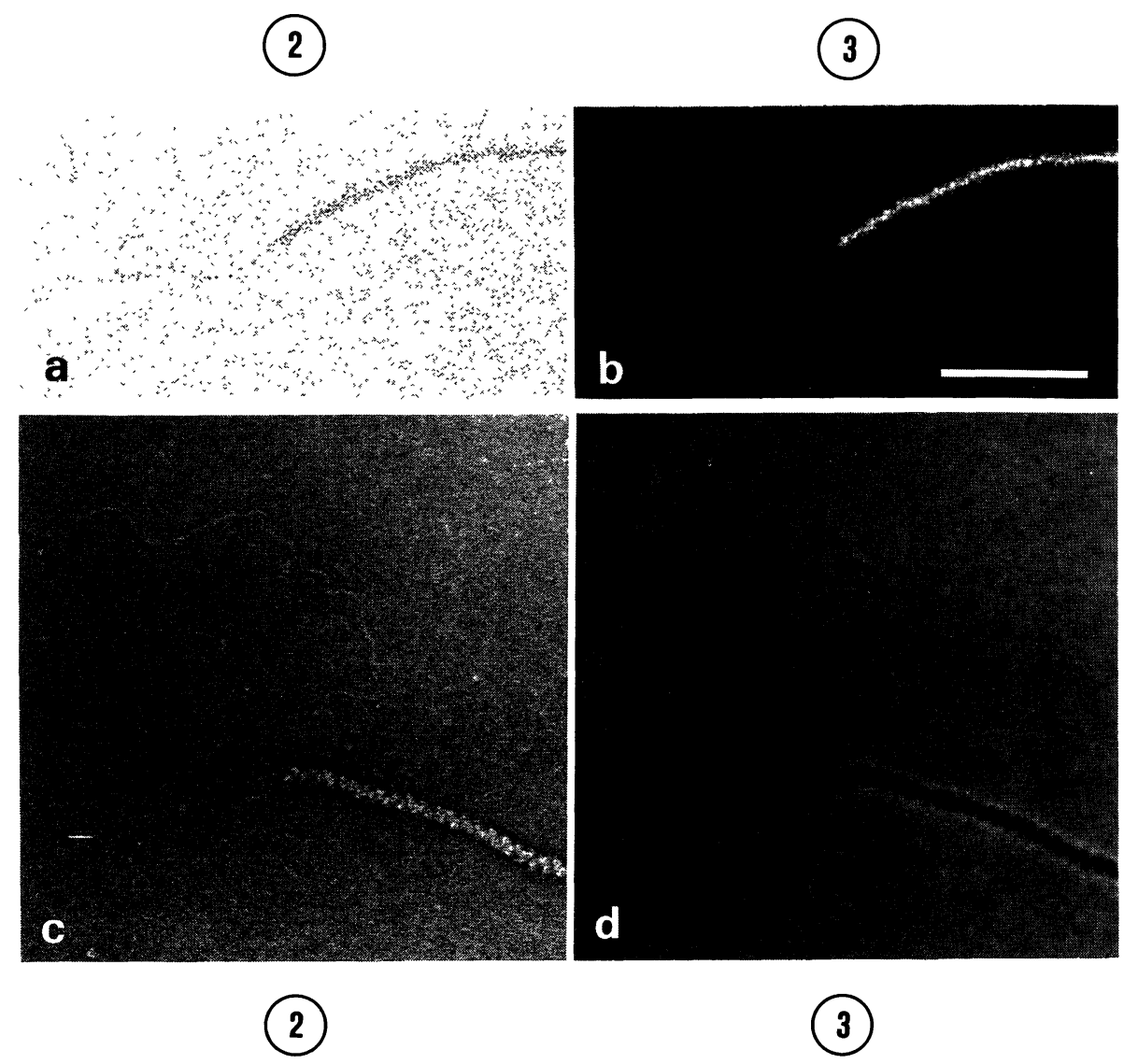

Fig. 4. - Positive (Figs. 4a and $4 \mathrm{~b}$ ) and negative (Figs. $4 \mathrm{c}$ and $4 \mathrm{~d}$ ) staining with uranyl acetate of recADNA complexes observed in imaging modes 2 (Figs. $4 \mathrm{a}$ and $4 \mathrm{c}$ ) and 3 at $\Delta E=114 \mathrm{eV}$ (Figs. $4 \mathrm{~b}$ and $4 \mathrm{~d}$ ). The visibility of positively stained molecules observed in bright-field image in poor. It is better in ESI with inelastic electrons corresponding to the electron energy loss peaks of uranium. The bright-field image of negatively stained DNA and recA-DNA is good, as well as its ESI counterpart. Bar $=0.1 \mu \mathrm{m}$.

ELECTRON MICROSCOPY. - Observations are performed with a Zeiss 902 TEM. For hollow cone illumination, the annular condenser aperture used is the Model B41 from Pelanne Instruments (Toulouse, France). This aperture fits well with a $40 \mu \mathrm{m}$ thin film objective aperture which stops all direct, non-scattered electrons. For spectroscopic imaging with parallel beam, the condenser aperture diameter is $400 \mu \mathrm{m}$ and the objective one of 100 or $40 \mu \mathrm{m}$. The spectrometer slit is usually settled at a width of about $20 \mathrm{eV}$. All pictures shown in this work have been recorded with a normal hairpin tungsten filament. Increased image quality due to higher electron density and beam coherence can be obtained with $\mathrm{LaB}_{6}$ filament.

PHOTO PRINTING. - In order to faithfully compare the relative contrast of pictures in the different modes, all pictures have been printed on Agfa Brovira-Speed grade 3, to keep the original differences of contrast. 


\section{Results.}

ClassificATION OF THE AVAILABLE IMAGING MODES. - For the used instrument, those modes are depending on the illumination type (determined by the shape of the condenser aperture) and on the adjustment of the spectrometer selection slit (without or with), see table I. Generally speaking, an image is called bright-field if it provides negative contrast, i.e. the scattering power of the material gives rise to a decreased intensity. On the contrary, a dark-field image exhibits positive contrast. A hole in a specimen will appear as bright or dark, in the bright- or dark-field images, respectively. In the present study, the different imaging modes labelled 1 to 6 have been used.

Table I. - Characteristics of the different imaging modes used with a Zeiss 902 and illustrated in figures 2 to 5 .

\begin{tabular}{|c|c|c|c|c|c|c|}
\hline $\begin{array}{l}\text { Illumination mode } \\
\text { Condenser aperture }\end{array}$ & \multicolumn{3}{|c|}{ Parallel } & \multicolumn{3}{|c|}{ Hollow cone } \\
\hline Spectrometer slit & no & yes & yes & no & yes & yes \\
\hline Selected electrons & total & elastic & inel & total & elastic & inel \\
\hline Resulting image & \multicolumn{2}{|c|}{ bright field } & \multicolumn{4}{|c|}{ dark field } \\
\hline Imaging mode & & & & & & \\
\hline
\end{tabular}

COMPARISON OF THE CONTRASTS OBTAINED WITH IMAGING MODES 1 TO 6.

Shadowed DNA (Fig. 2). - This is demonstrated on a sample of DNA rotary shadowed with platinum-carbon prepared according to the method A (Fig. 1). In bright-field, with all electrons, i.e. without the use of the spectrometer (Mode 1), the contrast is relatively weak, and furthermore depends on the defocus (Fig. 2a). A slight increase on contrast is obtained in Mode 2, where all inelastic electrons are eliminated (Fig. 2b). With the same parallel illumination, a dark-field image is obtained when inelastic electrons are selected (Mode 3), with increasing contrast and resolution for higher values of the selected energy loss (Figs. 2c-h). An optimum is obtained for $\Delta E$ values between $80 \mathrm{eV}$ (Fig. 2e) and $120 \mathrm{eV}$ (Fig. 2g), but useful images can be obtained up to $260 \mathrm{eV}$ (Fig. 2h), just below the carbon K-edge. As a matter of fact, the amount of electrons strongly decreases with increasing $\Delta E$ values, so that a compromise is to be found between the quality of the images (in term of contrast and resolution) and the exposition time (or dose) needed to take a picture, even with the help of a highly sensitive TV camera to focus the image. This optimum also depends strongly on the thickness of the sample. With shadowed DNA molecules, one must consider that the carbon film thickness is about 50-100 $\AA$, the molecule itself being $20 \AA$ and 
the metal deposit 10-15 $\AA$ as estimated from the width of the DNA molecule. Images of poorer quality are obtained with carbon films thicker than $100 \AA$ and even worst with thicker films like carbon-coated collodion which should be avoided.

Annular dark-field imaging without using the spectrometer (Mode 4, Fig. 2i) is very efficient since the current delivered by the condenser annular aperture (about $0.8 \mathrm{~mm}^{2}$ ) is 7.5 times higher than that selected by the $400 \mu \mathrm{m}$ (i.e. $0.125 \mathrm{~mm}^{2}$ ) condenser aperture used in ESI. It however provides a non homogeneous cross-over which does not allow good pictures below a 50,000 magnification, owing to the irregular intensity repartition (Figs. $2 \mathrm{i}$ and $2 \mathrm{j}$ ). Tiny bright points, more particularly visible on the molecules where the platinum deposit is thicker, correspond to diffracting metallic microaggregates of the shadowing material. A more contrasted image is obtained when elastically scattered electrons are selected (Mode 5), with an increase of the associated diffraction contribution (Fig. $2 \mathrm{j}$ ). In return, the selection of inelastically scattered electrons in the annular dark-field image (Mode 6) completely eliminates this drawback and provides very good images (Figs. 2k and 2l), similar to those obtained in Mode 3. Of course it is made difficult by its very low efficiency resulting in a small number of electrons on the fluorescent screen and the photo plate. It must be noted that only the geometry of the Zeiss 902 allows to perform energy filtering on the large angle scattered beam, although the new detector-spectrometer combination designed by Haider [20] for a STEM should also provide this opportunity.

Similar images can be obtained with molecules shadowed with a very thin layer of platinum or tungsten. With tungsten, the crystallization of the small clusters is reduced, no diffraction effects are encountered and the granularity of the metal deposit is lower. Other possibilities using chromium or platinum/iridium/carbon have also been used others [15] and are susceptible to benefit by ESI or energy filtered annular dark-field. It is important to note that the contrast increase is huge and thus allows imaging extremely thin metal coatings for which bright-field images do not provide sufficient contrast. Such thin shadows guarantee minimum alteration of the molecular details, which could be very important to localize tiny structures of biological importance at the molecular level. The recording of families of micrographs such as shown in figure 2 requires beam-resistent samples (not too thin carbon films and shadows), since a single field was irradiated for several minutes. In addition, this demonstrates the good stability of both sample and microscope.

Stained DNA. - Stained DNA molecules prepared following method B (Fig. 1) have also been imaged in the same six modes. It results in a very strong increase of contrast from bright-field (Modes 1 and 2) to dark-field images. In practice, among these modes, the filtered annular darkfield one (Mode 5) is chosen when very high doses of electrons are needed, especially when very small DNA molecules are observed at magnifications higher or equal to 85,000. In return, Mode 3 with $\Delta E$ in the order of $100 \mathrm{eV}$ provides very good images of larger DNA molecules or large fields containing numerous small molecules, which must be imaged at magnifications below 30,000 . This is illustrated in figures $3 \mathrm{a}-\mathrm{c}$ which show a molecule stained with uranyl acetate, imaged in Modes 2 , 5 and 6 (Figs. 3a, 3b and 3c, respectively). The contrast is very weak in elastic bright-field (Fig. 3a, Mode 2), very strong in annular dark-field (Figs. $3 \mathrm{~b}$ and $3 \mathrm{c}$ ), but the background is higher for the elastic image (Fig. 3b, Mode 5) than for the inelastic one (Fig. 3c, Mode 6). The comparison between the contrast and image quality obtained either with the elastically filtered annular darkfield (Fig. 3d, Mode 5) and with the usual ESI (Fig. 3e, Mode 3), is given for DNA molecules stained with thorium nitrate. The background of the ESI image is much smoother than that of the annular dark-field image.

Another example is given in figure 4 which shows complexes between DNA and the protein recA, a DNA-binding protein involved in the recombination process [21]. Depending on the 
pretreatment of the grid and on the thickness of uranyl acetate deposit on the grid, the molecules are either positively (method B) (Figs. 4a and 4b) or negatively (method C) (Figs. 4c and 4d) stained. In the former case, the DNA is rather difficult to see in the bright-field image (Fig. 4a, Mode 2), when it is better perceived in ESI with $\Delta E=114 \mathrm{eV}$ (Fig. 4b, Mode 3), which corresponds to the strong $\mathrm{O}_{4,5}$ peak of uranium. The helical coating of the recA protein on the DNA filament is not correctly resolved with such a thin deposit of uranium. When the DNA and the DNA-recA complex are embedded in a thicker layer of uranyl acetate which behaves as an amorphous film protecting the molecules (i.e. negative staining conditions), the helical structure of the polymerized recA, making a sleeve around the DNA, is clearly seen and the protruding naked DNA filament appears as a thin clear thread in bright-field (Fig. 4c, Mode 2). On ESI images at $\Delta E=114 \mathrm{eV}$ (Fig. 4d, Mode 3) areas where uranium accumulates are seen bright, the molecules being thus darker than the background. The fine structure of both the recA helice and the naked DNA molecules is clearly visible.

It must be noted that in ESI (Mode 3), there is very little phase contrast. Images must be recorded in the Gaussian focus condition and not slightly underfocused as in traditional brightfield. Finding this focusing condition may however be difficult, owing to the very reduced light available.

THE USE OF ESI FOR IMAGING ELEMENTAL DSITRIBUTIONS. - The EELS Atlas [22] shows that many edges can be used to provide element-specific images in ESI. In biology, many papers have been devoted to the specific localization of iron, calcium or phosphorus in sections of cellular tissues $[23,24]$, but very few have dealt with the chemical analysis of elements such as phosphorus or nitrogen on unstained molecules.

When applied to stained molecules with heavy metal ions (uranium, thorium, terbium...), the selection of the characteristic signal can be useful for improving the visualization of the object without dealing with the more complex problem of chemical mapping which requires extra data processing. The visualization of DNA molecules stained with or embedded into a film of uranyl acetate is optimal when the spectrometer is adjusted on the characteristic uranium core loss.

This is illustrated in figure 5 which shows a molecule positively stained with uranium salts. Optimum contrast is obtained when the uranium $\mathrm{O}_{4,5}$ peak $114 \mathrm{eV}$ is selected in the spectrometer slit (Fig. 5b). For DNA molecules stained with thorium, a similar optimum is obtained at about $100 \mathrm{eV}$. Comparable selective staining of DNA molecules has been obtained with lanthanides. Furthermore in some cases, it has been possible to use a mixture of thorium and lanthanides and to localize both elements on the DNA filament with adequate image filtering [25]. This leads to the eventual possibility of detecting specifically labelled zones of DNA, provided that specific labels can be built from sequence-specific compounds and selected metal-containing clusters or chelates.

\section{Discussion.}

For several reasons, the study of the conformation of molecules such as DNA constitutes an unusual challenge. The searched information is mostly of topological nature with the measurement of length, local curvature, etc... [26]. It may also be of chemical nature in the case of specific labelling. The specimen itsclf is very thin and made of light elements with very weak natural contrast. Furthermore, it is beam sensitive. Consequently one has to select the best combination of preparation technique and observation method in order to extract the required information from a contrast in the image at the desirable resolution level. Most biological studies necessitate the acquisition of images of numerous molecules (which means a great number of pixels in terms of 

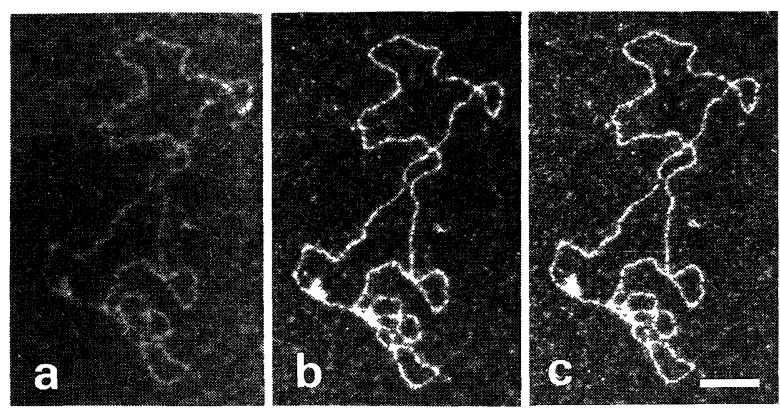

Fig. 5. - Electron Spectroscopic imaging of DNA molecules stained with uranium. Same material as for figure 5a-c have been taken in mode 3 with $\Delta E$ values equal to 90,114 and $140 \mathrm{eV}$, respectively. The optimum for contrast and resolution is obtained at the level of the peak of energy loss (Fig. 5b). Bar $=0.1 \mu \mathrm{m}$.

digitized images) rather than high resolution work over reduced image areas. This specificity excludes the routine use of the dedicated STEM, which does not allow easy observation of large and numerous fields, though its high intrinsic capabilities have been demonstrated for a selection of cases [27].

The three types of preparation methods schematized in figure 1 constitute three different solutions to observe the molecules: for shadowed molecules (A), the information is provided by mass-thickness variations of the metal on the specimen; in B (positive staining), the signal is also contained in the stain deposit which has been added to reveal it and it appears as an extra thickness and a local chemical change. Finally in $C$ (negative staining), the molecule by itself, embedded in the homogeneous stain film, provides the contrast as a local difference of composition averaged through the specimen. Though this latter solution constitutes the best way (apart cryomicroscopy) for preserving the biological objects, the other methods, which practically deal with "stained ashes", also indirectly convey the required information. Moreover, the decay of this information is much more rapid with electrons dose when using method $\mathrm{C}$ than the others.

In order to reveal changes of topographical and/or chemical parameters into detectable image intensity fluctuations, the nature of the involved contrast determines the choice of the operating mode with an energy selection microscope. In the present study, the major contrast generating mechanisms can be classified: (a) elastic scattering (or diffraction) at large angles occurs at random azimuthal angles when originating from a random distribution of very small metal aggregates; (b) inelastic scattering at small angles with non elemental-specific signals, i.e. mostly from the continuously decreasing background in the spectrum. It has been used mostly for the observation of thick specimens [28] but may also apply to the present situations to visualize mass-thickness variations through "contrast tuning" (see for instance: Reimer [29]); (c) characteristic chemical contrast contained in the detection of elements through their specific edge in the EELS spectrum (such as $\mathrm{O}_{4.5}$ for uranium at $110 \mathrm{eV}$ ).

For a given object, all of those contrast mechanisms contribute, at various levels depending on its nature: for instance, contrasts of type (a) and (b) are involved for shadowed specimens (Fig. 1A), while the three terms occur simultaneously for stained specimens (Figs. 1B and C).

The variety and diversity of operating modes offered by an ESI microscope, together with different illumination conditions, provide to the biologist user many solutions as demonstrated above. Mode 2, also called zero-loss filtering is used for contrast enhancement on plastic sections, and have been used also succesfully in cryomicroscopy [30]. However, modes 3 and 5 have shown to be of practical interest in biology. The first of these two modes is the inelastic one using a selected 
fraction of the EELS spectrum, and leading to a contrast-tuning (no characteristic edge included), or a chemical-selecting contrast (with a well defined edge included). It proves to be particularly fruitful for delivering images with high contrast and devoid of most speckles due to the diffraction contrast on individual metallic grains. It offers quite convenient images for a posteriori digital image recognition and automatic measurement processes [31]. The resolution is maintained at a high level as shown in the paper by Berger and Kohl [32], the spatial resolution increasing regularly with the value of the selected energy loss. The second one, mode 5 , is the elastically filtered annular dark-field mode. It provides images with high signal to noise ratio and good spatial resolution, because the blurring effect of the plasmon loss contribution is eliminated. On the other hand, it emphasizes the influence of the diffraction contrast.

\section{Conclusion.}

The present paper has dealt with non-conventional operating modes, at least in the biological field, for an energy filtering microscope derived from the early work of Castaing and Henry. The very fast development of such instruments in the recent years has been largely dedicated to analytical studies on sections, as it can be seen through the published proceedings of ESI microscope users [23-24]. If this latter field of application has shown all its interest, it seemed important to us to emphasize the other aspect concerning plain imaging and to discuss how the available information was conveyed and filtered through a superposition of dedicated specimen preparation techniques and selected contrast mechanisms.

\section{Acknowledgements.}

C. Colliex is thanked for his constant interest in the course of this interdisciplinary cooperation which has been fostered by the CNRS Ultimatech program. Financial support was provided by the "Ligue Nationale Française contre le Cancer", in addition to that of the CNRS, of the Gustave Roussy Institute and the "Association pour la Recherche contre le Cancer".

\section{References}

[1] BRACK C., DNA electron microscopy, Crit. Rev. Biochem. 10 (1981) 113-169.

[2] Dubochet J., Ducommun M., ZOLlinger M. and KellenBerger E., A new preparation method for dark-field electron microscopy of biomacromolecules, J. Ultrastruct. Res. 35 (1971) 147-167.

[3] Le Cam E., Theveny B., MignotTe B., Revet B. and Delain E., Quantitative electron microscopic analysis of DNA-protein interactions, J. Electron Microsc. Tech. 18 (1991) 375-386.

[4] BUSTAMANTE C., Direct observation and manipulation of single DNA molecule using fluorescence microscopy, Ann. Rev. Bioplyys. Biophys. Chem. 20 (1991) 415-446.

[5] CAIRNS J., The bacterial chromosome and its manner of replication as seen by autoradiography, J. Mol Biol. 6 (1963) 208-213.

[6] BLOOMFIELD V.A. and ARscotT P.G., Scanning tunneling microscopy of nucleic acids, Nucleic Acids Mol. Biol. 5 (1991) 39-53.

[7] LINDSAY S.M. and PHILIPP M., Can the scanning microscope sequence DNA? Genet. Anal. Tech. Appl. 8 (1991) 8-13.

[8] Bustamante C., Vesenka J., Tang C.L., Rees W., Guthold M. and Keller R., Circular molecules imaged in air by scanning force microscopy, Biochemistry 31 (1992) 22-26.

[9] Zenhausern F., Adrian M., Ten Heggeler-Bordier B., EmCh R., Jobin M., TABORElli M. and DESCOUTS P., Imaging of DNA by scanning force microscopy, J. Struct. Biol. 108 (1992) 69-73. 
[10] Delain E., Fourcade A., Coulaud D., Le Cam E., Barbin A., Poulin J.-C. and Revet B., Comparative observations of circular DNA in atomic force, tunnelling and electron microscopes, Nucleic Acids Res. submitted.

[11] BAUER R., Electron spectroscopic imaging: an advanced technique for imaging and analysis in transmission electron microscopy, Methods Microbiol. 20 (1988) 113-146.

[12] FOWLER W.E. and AEBI U., Preparation of single molecules and supramolecuar complexes for high resolution metal shadowing, J. Ultrastruct. Res. 83 (1983) 319-334.

[13] WALZTHÖNY D., EPPENBERGER H.M. and WALLIMANN T., Shadowing of elongated molecules (myosin, tropomyosin, collagen, and DNA) yields regular molecule-dependent heavy metal grain patterns, Eur. Cell Biol. 35 (1984) 216-225.

[14] Delain E., Thiery J.P., Coulaud D., Joliviere A. and HartMann L., Etude chimique et ultrastructurale de la glycoprotéine de Tamm et Horsfall ou uromucoïde, Biol. Cell. 39 (1980) 31-42.

[15] WEPF R., AMREIN M., BÜRKLI U. and GROSS H., Platinum/iridium/carbon: a high-resolution material for TEM, STM and SEM of biological macromolecules structures, J. Microsc. 163 (1991) 51-64.

[16] CASTAING R. and HENRY L., Filtrage magnétique des vitesses en microscopie électronique, C. R. Acad. Sci. Paris 255 (1962) 76-80.

[17] Delain E., POCHON F., BARRAY M. and VAN LEUVEN F., Ultrastructure of alpha 2-macroglobulins, Electron Microsc. Rev. 5 (1992) 231-281.

[18] REVET B., DELAIN E. and BAUER R., New possibilities in the observation of nucleic acids by spectroscopic imaging, and by direct image acquisition and treatment, Proc. XIth Int. Cong. on Electron Microscopy, Kyoto (1986) pp. 2409-2410.

[19] AEBI U. and POLLARD T.D., A glow discharge unit to render electron microscope grids and other surfaces hydrophilic, J. Electron Microsc. 7 (1987) 29-33.

[20] HAIDER M., A corrected double deflexion electron spectrometer equipped with a parallel recording system, Ultramicroscopy 28 (1989) 190-200.

[21] RocA A.I. and CoX M.M., The recA protein. Structure and function, Crit. Rev. Biochem Mol. Biol. 25 (1990) 415-456.

[22] AHN C.C. and KRIVANEK O.L., EELS Atlas. A reference collection of electron energy loss spectra covering all stable elements (Ed. Gatan, Inc., PA, 1983) p. 92.

[23] Proceedings of the European Workshop on electron spectroscopic imaging and analysis techniques, Ultramicroscopy 32 (1990) Special issue, pp. 1-91.

[24] Proceedings of the second Workshop on electron spectroscopic imaging, J. Microsc. 162 (1991) Special issue, pp. 1-200.

[25] MORY C. and COLliEX C., Elemental analysis near the single-atom detection level by processing sequences of energy-filtered images, Ultramicroscopy 28 (1989) 339-346.

[26] MUZARD G., THEVENY B. and REVET B., Electron microscopy of pBR322 DNA curvature. Comparison with theoretical models, EMBO J. 9 (1990) 1289-1298.

[27] MORY C., COllieX C., Revet B. and Delain E., Improved visualization of a single and double stranded nucleic acid by STEM, Ultramicroscopy 2 (1981) 161-168.

[28] COllieX C., MORY C., Olins A.L., Olins D.E. and TENCE M., Energy filtered STEM imaging of thick biological sections, J. Microsc. 153 (1989) 1-21.

[29] ReIMER L., Fromm I., HÚlK C. and ReNNEKAMP R., Energy-filtering transmission electron microscopy in materials science, Microsc. Microanal. Microstruct. 3 (1992) 141-157.

[30] SCHRODER R.R., HOFMAN W. and MENETRET J.F., Zero-loss energy filtering as improved imaging mode in cryoelectron microscopy of frozen-hydrated specimens, J. Struct. Biol. 105 (1990) 28-34.

[31] MASNOU S. and REVET B., unpublished results.

[32] BERGER A. and KOHL H., Elemental mapping using an imaging energy filter: image formation and resolution limits, Microsc. Microanal. Microstruct. 3 (1992) 159-174. 